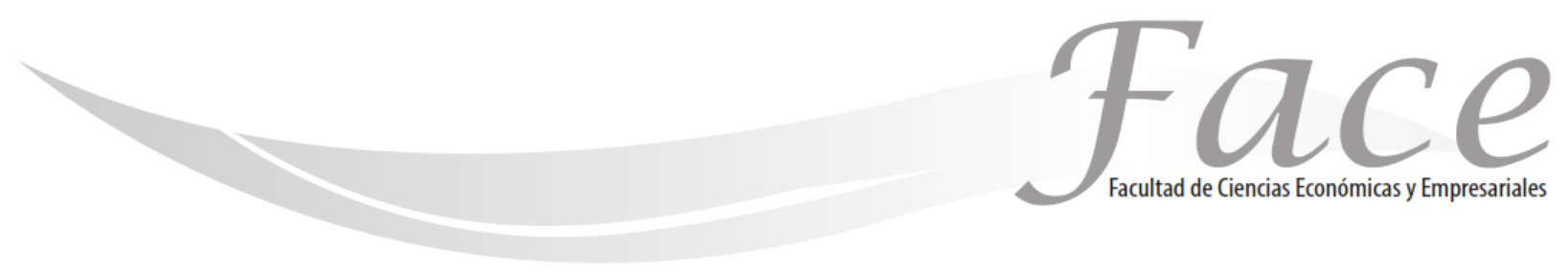

ISSN 1794-9920

Volumen $15-2$

Año 2015

Págs. 99 - 106

\title{
FILOSOFÍA GERENCIAL SEIS SIGMA EN LA GESTIÓN UNIVERSITARIA *
}

\author{
Mawency Vergel Ortega ** \\ José Joaquin Martinez Lozano ***
}

Fecha de Recepción: 29 de Junio 2015

Fecha de Aprobación: 20 de Octubre 2015

\begin{abstract}
Resumen:
El objetivo fundamental de la investigación es implementar la estrategia gerencial Seis Sigma en la gestión de procesos de la Vicerrectoría de Bienestar universitario de la Universidad Francisco de Paula Santander. La investigación sigue un enfoque cuantitativo de tipo campo nivel correlacional. Para el análisis e interpretación de la información se utilizan herramientas de diagramación, mapeo de procesos, análisis descriptivo y multivariado, jerárquico, estudios de capacidad, análisis de sistemas de medición, diseño de experimentos, pruebas de errores y cuadros de control. Una vez se implementa la filosofía seis sigma, se observa el mejoramiento continuo, mejoras en capacidad de procesos, efectividad del 99,9\% en variables de medición, mejora en percepciones de comunidad académica y administrativa, y estandarización de procesos, se evalúa el impacto de la aplicación de seis sigma en una unidad; promoviendo así, un flujo eficiente de sus productos y procesos.
\end{abstract}

Palabras claves: Gestión de procesos, estrategia gerencial, DMAIC-Seis Sigma; mejora; eficiencia; productividad; cero defectos.

\footnotetext{
* El artículo es producto del programa Sistemas de gestión de Calidad, proyecto Seis sigma en instituciones educativas de la línea de investigación Estadística y calidad - Grupo de investigación Euler - Centro de consultoría en Estadística de la Universidad Francisco de Paula Santander-Cúcuta Norte de Santander Colombia.

** Docente de la Universidad Francisco de Paula Santander - Cúcuta - Colombia. Ph.D en Educación, Magister en Educación Mención Gerencia EducacionalUniversidad Pedagógica Experimental Libertador -Venezuela. Contacto: mawency@ufps.edu.co

*** Docente de la Universidad Francisco de Paula Santander- Cúcuta - Colombia. Magister en Educación Mención Gerencia Educacional- Universidad Pedagógica Experimental Libertador -Venezuela. Contacto: Checo.j@gmail.com
} 


\title{
SIX SIGMA PHILOSOPHY IN UNIVERSITY MANAGEMENT
}

\begin{abstract}
:
The main purpose of the research was to implement the Six Sigma strategy in the process management of the University Francisco de Paula Santander's Welfare Vice-Rectory. The research was based on a quantitative approach of correlation type. The research was based on a quantitative correlation study approach. For the analysis and interpretation of the data the study used research tools such as diagramming and mapping process, descriptive and multivariate analysis, capability studies, systems measurement analysis, experiment design and error proofing. After the implementation of Six Sigma there was a substantial improvement in process capability, $99.99 \%$ effectiveness in measurement variables, and improvement in academic and administrative community perception and standardization process. The impact of Six Sigma was assessed; thus, promoting an efficient flow of products and process.
\end{abstract}

Keywords: Process Management, Planning, Management Strategy, DMAIC-Six Sigma, Improvement, Efficiency, Productivity, Cero Defect.

\section{FILOSOFIA GERENCIAL SEIS SIGMA NA GESTÃO UNIVERSITÁRIA}

\begin{abstract}
Resumo:
O principal objetivo da pesquisa foi analisar a utilização do Seis Sigma na gestão dos processos da Vice-reitoria de Bem-estar Universitário da Universidade Francisco de Paula Santander. A pesquisa baseou-se em um enfoque quantitativo de tipo correlação. As ferramentas utilizadas na análise e interpretação dos dados foram: diagramação e mapeamento de processos, análise descritiva e multivariada, estudos de capacidade, análise de sistemas de medição, desenho de experimentos, provas de erros e painéis de controle. Depois da aplicação do Seis Sigma observou-se um melhoramento contínuo nos processos de gestão, 99,99\% de efetividade nas variáveis de medição, melhora da percepção da comunidade acadêmica e administrativa e na padronização dos processos, avaliou-se o impacto da aplicação do Seis Sigma em uma unidade; promovendo assim, um fluxo eficiente de produtos e processos.

Palavras-Chave: Gestão de Processos, Planejamento, Estratégia Gerencial, DMAIC- Seis Sigma, Melhoria; Eficiência; Produtividade; Cero Defeitos.
\end{abstract}




\section{INTRODUCCIÓN:}

Como respuesta a las demandas de competitividad en un contexto de apertura e internacionalización de la economía, las organizaciones en América Latina comenzaron a asumir como estrategia gerencial la calidad total. Son muchas las compañías que utilizan la metodología DMAIC para la mejora de sus procesos. De hecho, de acuerdo a Demirkan, Spoher y Krishna (2011) la institucionalización del proceso DMAIC dentro de una organización lleva a desarrollar un gran número de personal con cinturones verdes y negros enfocados en iniciativas de mejora de procesos. Las instituciones educativas no ajenas a estos fenómenos organizacionales, se enfrentan también en un entorno de competencia $y$, deben afrontarlo en forma eficiente y efectiva, para cumplir con los objetivos del quehacer educativo.

Lo anterior, sin perder de vista la importancia de su impacto en el proceso educativo y en el desarrollo del país, lo cual implica la aplicación y desarrollo de estrategias de gestión que maximicen su impacto y sus costos, conlleva además, el rediseño y mejoramiento de los procesos organizacionales de las unidades académico administrativas para su óptimo uso en el cumplimiento de sus objetivos misionales.

Esta interiorización de filosofías de gestión modernas no ha sido un proceso fácil y la Universidad Francisco de Paula Santander no ha sido ajena a este fenómeno, enfrentaron problemas característicos de organizaciones que van creciendo sin una orientación clara en su proceso de gestión. Sin embargo, los directivos conscientes de la necesidad de cambiar el estilo de gestión y de adelantar procesos de mejora en el quehacer de su área implementaron sistemas de gestión de calidad y estrategias a través de seis sigma.

El objetivo de la investigación tuvo como propósito implementar la estrategia gerencial Seis Sigma en la gestión de la Vicerrectoría de Bienestar universitario. Su importancia radica en el mejoramiento continuo de procesos gerenciales en administrar y operar la unidad de manera eficaz, con el menor costo, es decir el impacto de la aplicación en el flujo eficiente de sus procesos. Los objetivos específicos planteados buscaron diagnosticar la situación actual que presenta la gestión de los procesos de la vicerrectoría de Bienestar universitario, determinar la factibilidad económica, administrativa técnica y legal de implementar la filosofía seis sigma, implementar el modelo Seis Sigma en el proceso de gestión para facilitar la mejora continua y evaluar el impacto de la aplicación y desarrollo de la metodología Seis Sigma. De esta manera, la investigación fue pionera en implementar esta filosofía gerencial dentro de los procesos de impacto en la comunidad educativa lo cual le permitiría servir de modelo para otros procesos en Instituciones educativas del país.

\section{METODOLOGÍA:}

El estudio estuvo enmarcado en el enfoque cuantitativo, apoyado en un estudio de tipo campo, documental siguiendo un nivel correlacional (Hernández, Fernández y Baptista, 2003); el cual, con base en diversas concepciones, se desarrolla a través de etapas como diagnóstico de necesidades, planteamiento y fundamentación teórica de la propuesta; procedimiento metodológico, actividades y recursos necesarios para su ejecución y el análisis de viabilidad tanto económica, financiero, social y la posibilidad de su implementación (Arias 2012; Álvarez, 2002).

Las herramientas y el enfoque según Lean Six Sigma México (2011) busca ante Seis Sigma persigue la mejora de los procesos aunque en un sentido más amplio y menos definido a priori, calidad, eficiencia, niveles de servicio, hace uso extensivo de los datos para entender el comportamiento de los procesos e identificar mejoras. Se aplicaron como instrumentos listas de cotejo, encuestas, entrevistas a personal administrativo, personal docente y estudiantes. Caracterizada la población y servicios se realiza validez de instrumentos antes de implementar seis sigma cuya fiabilidad fue de 0,89 e índice de kappa igual a 0,9 . En la etapa de diagnóstico se utilizó modelo tipo $C$ de Kaufman (Kaufman y Monteiro, 2004), para establecer prioridades, se identifican los posibles proyectos Seis Sigma, que deben ser evaluados.

La segunda fase de la metodología, medición, consiste en la caracterización del proceso identificando necesidades clave de la comunidad académica, las características de los servicios (o variables del resultado) y los parámetros (variables de entrada) que afectan el funcionamiento del proceso. Los resultados, el análisis documental y económico financiero, llevaron a la identificación y selección de problemas prioritarios a resolver en procesos, así como a medir el desempeño de la gestión unidades adoptando el modelo de seis sigma en la gestión (Beltrán, 2012; Casanova, 2005; Iscea Latin America, 2011). En la tercera etapa, análisis, el equipo analiza los datos de resultados actuales e históricos. Se desarrollan y comprueban hipótesis sobre posibles relaciones causaefecto utilizando las herramientas estadísticas pertinentes. 
La capacidad según Brue y Lansbuy (2006), el cociente entre la diferencia de los límites superior e inferior y seis veces la desviación, y límite superior igual a probabilidad de éxito más el rango por la confiabilidad se obtiene según López (2011), la capacidad de los procesos. Análisis de cumplimiento de procesos permite determinar el número de defectos por millón de oportunidades de solución de los problemas para cumplimiento con parámetros de calidad y eficiencia de los procesos (Pande y Newman, 2002). Se siguen parámetros establecidos en metodología utilizada por seis sigma, se estipula escala para determinar si el proceso está en estado satisfactorio, adecuado 0 inadecuado (Wheat y Mills, 2004).

La conformación de equipos de mejora y selección de proyectos a ejecutar requiere realizar un análisis causa efecto y estimación de tiempos para ejecutar proyectos (Medrano y Vergel, 2006). De esta manera, los equipos inician entonces la etapa de planificación. Para ello, se utiliza matriz de marco lógico (Ministerio de Administraciones Públicas, 2003), se elabora árbol de problemas, de acuerdo a resultados estadísticos sobre causas de satisfacción del servicio, indicadores evaluados y lluvia de ideas.

De otra parte, análisis comparativos después de implementada la filosofía permiten evaluar resultados de su aplicación y desarrollo. Teniendo en cuenta directrices en torno a la política de calidad, se asumen como directrices un capital humano competente, cumplimiento de requisitos legales, cumplimiento de propósitos de la institución, estructura organizacional dinámica, desarrollo de procesos eficientes, eficaces y efectivos, mejoramiento continuo, satisfacción de usuarios, cumplimiento de normas y parámetros establecidos por el estado.

Tabla $N^{\circ} 1$

Cumplimiento Dimensiones formación, social.
En la fase de mejora se establecen acciones para cumplimiento de los objetivos de calidad, plan estratégico y de acción para implementar en menos de seis meses, con el fin de comparar resultados antes y después de implementada la metodología utilizada. Se traza además como perspectivas a tener en cuenta en el control, las perspectivas financiera, clientes, procesos y perspectiva recursos humanos potenciales (Vergel, 2011). La última fase, control, consiste en diseñar y documentar los controles necesarios para asegurar que la mejora en la capacidad de los procesos se mantiene una vez se implementan mejoras.

La población estuvo constituida por 13.143 estudiantes (11.685 presenciales y 1.458 a distancia); 741 docentes; y 280 administrativos. Se realizó muestreo probabilístico estratificado por asignación proporcional con un $95 \%$ de confiabilidad, se aplicaron instrumentos a 122 profesores: 20 profesores tiempo completo, 102 profesores cátedra; 40 administrativos (16 de planta, 24 contratistas); y 372 estudiantes modalidad presencial (13 ingeniería electromecánica, 26 civil, 12 mecánica, 20 sistemas, 14 electrónica, 11 minas, 5 producción industrial, 12 industrial, 1 tecnología electromecánica, 8 obras civiles, 6 tecnología química, 49 administración, 49 contaduría pública, 23 contaduría nocturna, 19 administración nocturno, 10 licenciatura en Matemáticas, 15 licenciatura en Biología, 14 arquitectura, 5 producción biotecnológica, 10 ingeniería biotecnológica, 4 producción agrícola, 5 agronómica, 5 ingeniera de producción animal, 4 pecuaria, 11 producción agroindustrial, 9 Ingeniería agroindustrial, 4 tecnología agropecuaria, 7 de enfermería).

\section{RESULTADOS:}

El análisis (Tabla $\mathrm{N}^{\circ} 1$ ), muestra 2 indicadores de impacto crítico en la dimensión Liderazgo con defecto, en la dimensión comunicación indicadores canal de comunicación y comunicación interna presentan defectos, mientras canales en reuniones se muestran aceptables.

\begin{tabular}{|c|c|c|c|c|c|}
\hline & Indicadores & $\%$ & varianza & Impacto & Atributo \\
\hline \multirow{5}{*}{ 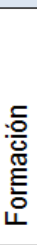 } & $\begin{array}{l}\text { Perfil, Disponibilidad tiempo Desarrollo aptitudes, logros, aporte a } \\
\text { formación integral }\end{array}$ & 100 & 0 & significativo & Cumple \\
\hline & Programas ofertados Desempeño & 80 & 0.4 & sustituible & Aceptable \\
\hline & Satisfacción servicio, Dotación, infraestructura & 80 & 0.4 & crítico & Defecto \\
\hline & Proyectos de investigación Grupos de investigación & 0 & 1 & significativo & Inaceptable \\
\hline & Desviación dimensión & & 0.316 & & \\
\hline \multirow{5}{*}{ 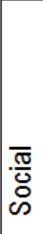 } & Número estímulos, becatrabajo, monitoría & 100 & 0 & sustituible & Cumple \\
\hline & Número acceso almuerzo, beneficiario por estrato, eventos & 80 & 0.3 & sustituible & Aceptable \\
\hline & costo servicio médico, tiempo de atención, \% conforme servicio & 30 & 0.488 & significativo & Defecto \\
\hline & $\begin{array}{l}\text { Acceso servicios, servicio médico, participación en toma de } \\
\text { decisiones }\end{array}$ & 20 & 0.4 & crítico & Inaceptable \\
\hline & Desviación dimensión & & 0.361 & & \\
\hline
\end{tabular}

Fuente: Autor 
En dimensión control los indicadores evaluación, control de documentos, auditoria Interna, mapa de riesgos, acciones correctivas son críticos e inaceptables. En lo referente a gestión de personal, 7 indicadores fueron considerados críticos, 3 inaceptable y 3 aceptable. En proceso mercadeo, 12 indicadores se consideran críticos e inaceptables. Seis indicadores fueron evaluados como críticos en la dimensión planificación, a saber, normas, tareas, toma de decisiones ( $\mathrm{z}=0$ a $\mathrm{z}=-1,82$; impacto significativo, atributo: cumple), control, competencia, cumplimiento misión, criterios beca-trabajo-monitoria $(\mathrm{z}=0 \mathrm{a}$ $z=-0,4$; impacto significativo, atributo aceptable), estrategia, cumplimiento a 2012 del plan, plan, cumplimiento misión, plan mejoramiento ( $z>2$; crítico); tiempo ejecución (defecto). Dimensión liderazgo muestra en tipo de líder (cumplimiento alto, perfil, metas, toma de decisiones, delegación de autoridad con impacto significativo, aceptable), delegación de autoridad intrascendente.
El equipo de mejora, consideró que el problema central correspondía a baja calificación obtenida en la gestión de

Bienestar universitario en 2012 (3.6) referente a asistencia médica, sistema de inclusión en cursos de formación integral, deficiencias en sistema de quejas institucional. Se encontraron 192,3 defectos por millón de oportunidades, detectando un porcentaje de calidad de 99,97\%, es decir, es un proceso cinco sigma.

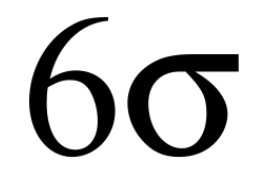

Tabla $\mathrm{N}^{\circ} 2$.

Defectos por millón de oportunidades y valor z sigma 2013

\begin{tabular}{|l|r|r|r|c|}
\hline \multicolumn{1}{|c|}{ Dimensiones } & $\begin{array}{c}\text { Defecto por } \\
\text { unidad }\end{array}$ & $\begin{array}{c}\text { Defecto por } \\
\text { oportunidad }\end{array}$ & $\begin{array}{c}\text { Defecto por millón de } \\
\text { oportunidades }\end{array}$ & Sigma store \\
\hline Planificación, social, servicios & 0.818 & 0.833 & 833333.36 & 0.5 \\
\hline Liderazgo & 0.2818 & 0.26 & 263157.9 & 2 \\
\hline Formación, financiero & 0.461 & 0.9 & 900000 & 0.5 \\
\hline Recursos & 0.818 & 1 & 1000000 & 0 \\
\hline Personal & 0.46 & 0.4666 & 466666.7 & 1.6 \\
\hline Comunicación, control, mercadeo & 1 & 0.3 & 300000 & 2 \\
\hline
\end{tabular}

Fuente: Autor

Figura $\mathrm{N}^{\circ} 1$.

Capacidad, mapa de control Gestión

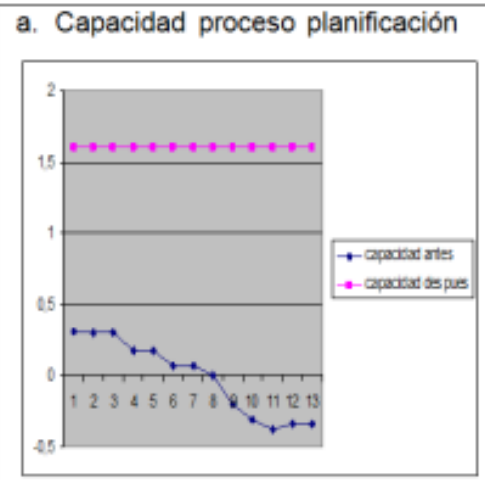

Enero 2013- Marzo 2014
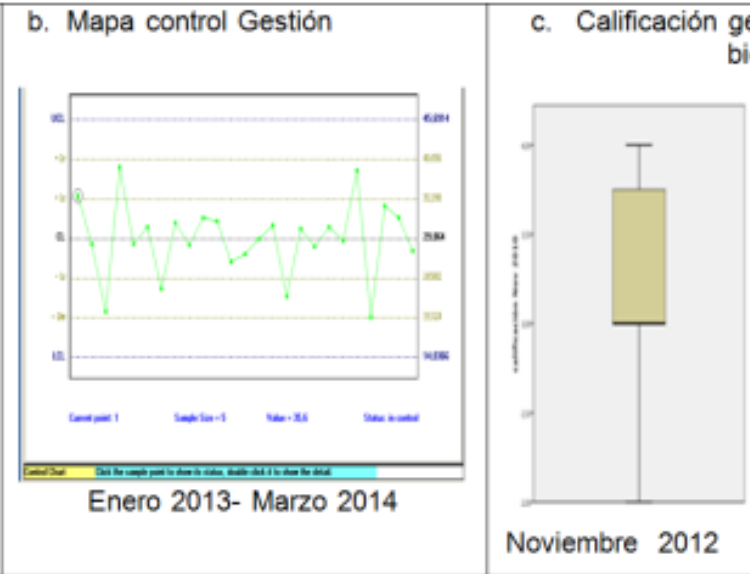

Noviembre 2012 gestión lider vicerectores bienestar

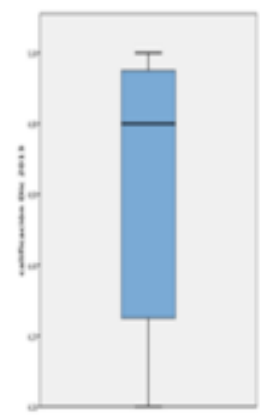

Marzo 2014

Fuente: Autor 
Implementado seis sigma, se resalta la disminución de la variabilidad del proceso planificación (Figura 1), incluso en picos observados en gráfico antes de implementar seis sigma (azul), lo cual indica tendencia a mantener índices de cumplimiento dentro de lo normal, con calidad superior a $90 \%$. Se observó necesidad de establecer canales de divulgación de información veraz de problemáticas existentes, funciones de cada unidad. Con un $99,9 \%$ de confiabilidad el promedio de calificación en puntaje de gestión mejoró implementada la filosofía, obteniendo puntajes entre 4 y 5 en 2014.

En aceptabilidad del sistema de gestión, la comunidad manifestó ventajas a la hora de trabajar por procesos, teniendo en cuenta visión sistémica, según concepción Zambrano (2013) mejora aspectos financieros, trabajo en equipo por unidades, organización y agilidad, software, estandarización, se integra a los procesos de la Universidad, seguimiento, evaluación, mejoramiento continuo, calidad en la prestación de servicios y apropiación de estos, mejor cobertura de la población, ampliación servicio nocturno y sábado positivo, actividades niños de comunidad, convenios e implementación de sistemas de información, mejora y publicación de eventos, plan comunicacional, gratuidad de carnet, ampliación horarios, oferta cursos, mejora en web, redes sociales y plataforma para egresados.

\section{DISCUSIÓN:}

Seis Sigma, según López (2011), es un enfoque revolucionario de gestión que mide y mejora la calidad. $\mathrm{Ha}$ llegado a ser un método de referencia para satisfacer las necesidades de los clientes y lograrlo con niveles próximos a la perfección, en la investigación se evidenció que el enfoque puede ser implementado en una institución de educación superior, en sus procesos, unidades, y productos, dando respuesta a necesidades de la comunidad académica.

Diseño, comunicación, formación, producción, administración, pérdidas pueden mejorar a través de esta filosofía. Para Pérez y García (2014), las posibilidades de mejora y de ahorro de costos son enormes, pero el proceso Seis Sigma requiere el compromiso de tiempo, talento, dedicación, persistencia y, por supuesto, inversión; en la vicerrectoría de bienestar, el incrementar indicadores e implementar sistemas de información tuvo rechazo en unidad deportes, control de asistencia a clases y oferta de cursos según necesidades de estudiantes causó incomodidad en Jefe de División de servicios asistenciales. No obstante, existió mejora en satisfacción de docentes, estudiantes y personal administrativo en efectividad de servicios de todas las unidades.

La determinación de la capacidad de los procesos, demuestra que puede cumplir con la estimación de la demanda de servicios y necesidades de la comunidad. El principal problema en las causas externas generalmente en producción es la falta de material (Álvarez, 2012; Centro Nacional de Productividad, 2014; Zambrano y Miranda, 2013), lo que en muchas ocasiones es el resultado de los trámites requeridos para adjudicar una compra producto de tramitología; en el caso de procesos de bienestar universitario el principal problema se centra en tiempos de respuesta a necesidades, procedimientos en especial en torno a inscripciones, inicio de cursos y seguimiento y control de los mismos.

Para Aditec Ingenieros (2011) la implementación de las mejoras (entrenamiento al personal de mantenimiento y de la línea, así como la creación del plan de mantenimiento) logra mayores ingresos anuales, al lograr cubrir la demanda establecida. Al igual que lo manifiesta Ocampo y Pabón (2012) se logra en la vicerrectoría de bienestar universitario, una mejora significativa por la correcta implementación de planes adecuados en los procesos, al incorporar al análisis aspectos lógicos, matemáticos y estructurales de los sistemas y procesos siendo simulados para la deserción, servicios, proyección de estudiantes y docentes en cursos, programas y estímulos, previendo necesidades, ajustando y renovando planes y oferta, a través de modelos simulados.

La percepción de la calidad de servicio mejoró para el líder, jefes, procesos y servicios. Los costos disminuyeron y el número de beneficiarios aumentó. No obstante, la mejora de seis sigma no se alcanza en todos los procesos, pero se obtiene cinco sigma lo cual indica que aún hay bastante por mejorar en cuanto a eficiencia de procesos.

\section{CONCLUSIONES:}

La implementación de seis sigma en una unidad de una institución universitaria permite mejorar la capacidad del proceso de planificación, así mismo reducir la variabilidad del mismo y muestra la correlación entre satisfacción del cliente y la calificación de la gestión, así como los logros en el sistema de gestión de calidad al reducir tiempos.

En el Bienestar de la Universidad Francisco de Paula Santander, existió un incremento significativo de estímulos orientados a estudiantes de bajos recursos, población 
desplazada, víctimas de la violencia, así como estímulos en beca trabajo, monitorias, becas por participación en eventos y otros estímulos estipulados en Acuerdos de la universidad, se aumentó la capacidad del proceso en cada uno de sus indicadores, observándose tendencia a la estabilidad del mismo.

El plan de mejora incorporó plan de mejora en infraestructura, diseñándose planos arquitectónicos de oficina y edificio con infraestructura ideal conectada a medio ambiente, así como mejora en cursos a ofertar, incorporación de egresados (apoyo universiatrabajando.com), mejora en plataforma a través de cursos virtuales.

De otra parte, mejora en el clima organizacional dentro de cada unidad, al descentralizar el proceso y permitir toma de decisiones donde intervienen todos los miembros de un equipo con características particulares en formación. El apoyo desde la dirección de la vicerrectoría, no se considera algunas veces aceptada por pérdida de liderazgo de cada jefe, sin embargo, mejora los indicadores de manera significativa y el jefe que entiende la implementación de metodología mejora indicadores y aceptabilidad en población de estudiantes. La implementación de metodología permite sustentar las decisiones a través de proceso investigativo utilizando herramientas estadísticas y pruebas de hipótesis sobre datos objetivos y precisos. Utiliza una estructura para la detección y solución de los problemas y enfoque proactivo, en el cual se observa la causa raíz del problema para llegar a soluciones sólidas y prevenir la repetición.

Sin embargo, es indispensable orientar los equipos buscando lograr objetivos y no categorizarles de acuerdo a resultados, así mismo el control del liderazgo en la toma de decisiones es un factor algunas veces difícil de manejar, pero bajo el apoyo de la unidad de orientación o presencia de un psicólogo en los equipos, es posible llegar a acuerdos y sustentar decisiones en la toma de datos y estudio de causas y consecuencias de la no ejecución de una tarea o proyecto.

Seis Sigma interviene en la cultura de una organización dentro de una institución universitaria. En este tipo de organizaciones el verdadero triunfo es sostener el cambio, lograr que el cambio perdure, y se implemente como un sistema gerencial, que se utilice y sirva a los fines de la organización, esto, utilizando herramientas de control que permitan monitorear logros, transparencia y avances en la mejora de un proceso.

La metodología Seis Sigma aparece como una potente estrategia para lograr importantes mejoras en los procesos de planificación (Craig, 2005; Pérez y García, 2014). Es así como al implementar seis sigma en la Vicerectoría de Bienestar universitario, aumenta la capacidad de 0,5 a 1,6, es decir, el proceso pasa de un estado inapropiado a un estado satisfactorio. De igual manera disminuye la variabilidad del proceso, mostrando eficiencia en manejar índices de calidad dentro de lo normal.

Así mismo, tiene como característica distintiva el desarrollar proyectos de mejora en plazos de corta duración y el realizar la evaluación de sus resultados en términos de capacidad y económicos, aumentan la posibilidad de implementación en el ámbito universitario.

De otra parte, el incentivar y promover la participación de los colaboradores al interior de la vicerrectoría es fuente de creatividad e innovación constante, la misma que genera beneficios económicos, sociales y ambientales, a través del trabajo en equipo, la toma de decisiones estructurada, soportada en los datos y el uso de herramientas estadísticas.

Una cultura de mejoramiento continuo de los procesos promueve la competitividad y el desarrollo de la vicerrectoría, haciéndola cada vez más eficiente, desarrollando ventajas y diferenciación. El Sistema Seis Sigma es aplicable tanto a procesos técnicos como a no técnicos. En un proceso técnico existen oportunidades para recolectar datos y tomar decisiones; en cambio en un proceso no técnico es muy difícil de visualizarlos, porque carece de procesos administrativos, más aún estos procesos requieren ser tratados como sistemas para entenderlos mejor y así discernir los errores y fallas.

La evaluación del impacto financiero real de un Equipo y el efecto real no financiero conseguido por el son aspectos diferentes. La dirección debe tener en cuenta factores a la hora de asignar un problema a un equipo, estos factores son, tiempo a lo largo del cual nos podremos beneficiar de las ventajas que se consigan, número de unidades que se beneficiarán de la mejora, potencial de mejora a priori, posible extensión de lo aprendido a otros ámbitos, facilidad para transformar las ventajas en ahorros reales.

La elección de los temas o áreas de mejora es un elemento crítico al que hay que dedicar el tiempo suficiente. En momentos en que sea necesario demostrar la rentabilidad de los equipos (señal de existencia de resistencia activa) debe elegirse problemas cuyos efectos sean medibles de modo objetivo y aceptado por todos los implicados. 


\section{REFERENCIAS:}

Aditec Ingenieros. (2011). Modelado, simulación y optimización. Recuperado de http://www.aditecingenieros.com/arena1. html

Álvarez, M. (2002). Aplicación de la metodología Six sigma en los procesos de manufactura de la planta envasadora de lubricantes Cardon. Tesis de Maestría, Universidad Nacional experimental del Táchira, San Cristóbal, Venezuela.

Arias, G. (2012). Tesis y proyectos de investigación. 2ed. Caracas: Episteme.

Beltrán, J. (2012). Guía para una gestión basada en procesos. Sevilla, España: Instituto Andaluz de Tecnología.

Bernal, H. (1992). La filosofía de la Calidad Total aplicada a la educación: Un manual de reflexión sobre el tema. Bogotá: Violeta.

Brue, G. \& Launsby, R. (2006). Desing for Six Sigma. New York: McGraw Hill.

Casanova, R. (2005). Mejoramiento de los tiempos operativos a las llaves hidráulicas basado en la metodología six sigma en Areke Energy C.A. Tesis de Maestría, Universidad Gran Mariscal de Ayacucho, Barcelona, España.

Centro Nacional de Productividad. $(01,04,2014)$. ¿Qué es Seis Sigma? Recuperado de: http://www.cnp.org.co

Craig, G. (2005). Six sigma for Dummies. Canadá: Wiley Publishing.

Dermikan, H., Spoher, J. \& Krishna, V. (2011). Service System Implementation. New York: Springer Publishing.

Hernández, R., Fernández, C. \& Baptista, P. (2003). Metodología de la Investigación. México: McGrawHill.

Iscea Latin America. (2011). Certified supply chain manager. México: Iscea.

Kaufman, D. \& Monteiro, M. (2004). Programas de qualidade seis sigma - característica distintivas do modelo dmaic e dfss. Recuperado de: http://www.leansixsigma.com.br/acervo/acervo_391 12939.pdf
López, C. (2011). La metodología Seis Sigma ¿qué es? ¿para qué sirve? ¿cómo se aplica? ¿requerimientos para su implementación? ¿etapas de implementación?. Recuperado de: http://www.gestiopolis.com/ recursos/experto/catsexp/ pagans/ger/no12/6sigma.htm

Medrano, C y Vergel, M. (2006). Evaluación del programa de Licenciatura en Matemáticas e informática de la Universidad Francisco de Paula Santander. Tesis de Especialización, Universidad Francisco de Paula Santander, Cúcuta, Colombia.

Ministerio de Administraciones Públicas (2003). Modelo EFQM de Excelencia. Recuperado de: http://www.map.es/iniciativas/mejora_de_la_administ racion_general_del_estado/servicios_publicos/evalua cion_calidad/calidad/programas_basicos/pg_evalua/d ocumento_efqm/efqm_criterios/document_es/Modelo _EFQM_202003_CRITERIOS.pdf.[Consulta: 2011, Agosto 24]

Ocampo, J. \& Pavón, A. (2012). Integrando la Metodología DMAIC de Seis Sigma con la simulación de Eventos Discretos en Flexsim. Recuperado de: http://www.laccei.org/LACCEI2012Panama/RefereedPapers/RP147.pdf

Pande, S. \& Neuman, P. (2002). Las claves prácticas de Seis Sigma. Una guía dirigida a los equipos de mejora de procesos. Bogotá: McGraw Hill.

Pérez, E. \& García, M. (2014). Implementación de la metodología DMAIC-Seis Sigma en el envasado de licores en Fanal. Revista Tecnología en Marcha. 27 (3), 88-106.

Vergel, M. (2011). Modelo gestión Vicerrectoría de Bienestar universitario de la Universidad Francisco de Paula Santander. Tesis de Maestría, Universidad Pedagógica Experimental Libertador, Rubio, Venezuela.

Wheat, B. y Mills, C. (2004). Seis Sigma. Una parábola sobre el camino hacia la excelencia y una empresa esbelta. Bogotá: Norma.

Zambrano, M. \& Miranda, M. (2013) Diagnóstico de las finanzas del Municipio de San José de Cúcuta 20032011. Revista Face, 4 (1). 11-35. 